\title{
The visible hand behind properties
}

\author{
Zibin Chen ${ }^{1}$, Xiaozhou Liao ${ }^{1}$, Jun Chen ${ }^{2}$, Shujun Zhang $^{3}$ \\ ${ }^{1}$ School of Aerospace, Mechanical \& Mechatronic Engineering, University of Sydney, NSW 2000, Australia. \\ ${ }^{2}$ Beijing Advanced Innovation Center for Materials Genome Engineering, and Department of Physical Chemistry, University of \\ Science and Technology Beijing, Beijing 100083, China. \\ ${ }^{3}$ Institute for Superconducting and Electronic Materials, Australian Institute for Innovative Materials, University of Wollongong, \\ NSW 2500, Australia.
}

Correspondence to: Prof. Shujun Zhang, Institute for Superconducting and Electronic Materials, Australian Institute for Innovative Materials, University of Wollongong, NSW 2500, Australia. E-mail: shujun@uow.edu.au

How to cite this article: Chen Z, Liao X, Chen J, Zhang S. The visible hand behind properties. Microstructures 2021;1:2021001. https://dx.doi.org/10.20517/microstructures.2021.01

Received: 22 Mar 2021 Accepted: 22 Mar 2021 Available online: 26 Mar 2021

Academic Editor: Shu-Jun Zhang Copy Editor: Xi-Jun Chen Production Editor: Xi-Jun Chen

Designing new materials with desirable properties has been tirelessly pursued by human beings for centuries. From the discovery of using bronze for weapon forging in the Bronze age to the fabrication of multifunctional graphene with remarkable properties in the information age, human beings have gone through a very long learning process from trying (trial-and-error) to designing (knowing why and proceeding with purpose). The sharp turn of such change, however, came only in the last century when people realized that there is a visible hand behind the materials - microstructure - through which the properties of materials can be manipulated.

Microstructure, which is the nature, dimensions, quantity, and distribution of structural elements or phases that make up the materials, determines the properties of materials. Understanding microstructure-property relationships is critical for the design of materials. In structural materials, microstructures, from microscale grain morphology and crystallographic orientation to atomic scale short range ordering, determine every aspect of mechanical properties. While in functional materials, microstructures with different dimensions, shapes, forms, phases, and compositions are major attributors to their electrical, magnetic, optical, thermal, and chemical properties. 
However, it is not always easy to understand microstructure. At least, we need to see it before understanding it. The smallest dimension of an object that the human eye can differentiate is about $0.1 \mathrm{~mm}$. With this length scale, it is impossible to investigate the "actual" structures in materials. Thanks to the development of optical microscopy in the 17th century, humans have been able to see incredibly small things impossible to see with the naked eye. Since the 20th century, modern characterization techniques, including X-ray diffraction (synchrotron X-ray), neutron scattering, electron microscopy, scanning probe microscopy, atom probe tomography, and spectroscopy (such as Raman and Infrared spectroscopy, mass spectroscopy, electron dispersive spectroscopy, electron energy loss spectroscopy, etc.), have been developed to allow the investigation of microstructures from the microscale to the atomic scale. The most recent development in in-situ technologies even allows real-time observation of the microstructural evolution of materials under external stimulations, including thermal stimulation in a broad temperature, electric field, static/dynamic stress, and magnetic field, enabling the complete understanding of microstructure kinetics and the underlying mechanisms of the microstructure-property relationships. Revealing structure-property relationships of materials will turn the visible hand into a powerful tool for controlling and manipulating material properties.

Microstructures are not only important to the field of inorganic materials, but also beneficial to the field of biology where organic materials are the major subjects of investigation. X-ray crystallography and recently developed revolutionary cryo-microscopy techniques allow the structural characterization of organic materials, including cells and proteins, with ultra-high resolution. This provides a profound understanding of life-related contents.

Characterization of microstructures is never the end game for scientists and engineers. Further improving the capability of predicting and designing microstructures is always in their wish list. Based on experimental parameters and theoretical approximation, various microstructure and property modelling techniques, including first principles calculation, molecular dynamics, Monte Carlo simulation, and phase field modelling have been developed to predict microstructures and guide the fabrication and design of materials from the fundamental level. The combination of theoretical calculation and experimental observation provides a perfect match to fully understand and manipulate the microstructures in material design.

The chain of design-fabrication-microstructure-property-application plays the key role in materials research and development, where the understanding and utilization of microstructure can speed up and benefit the design of new materials, and this in turn will be the driving force for the exploration of new phenomena and smart structures/devices at the microscale and nanoscale. We strongly believe that the new journal Microstructures will benefit the communities of physicists, chemists, biologists, and engineers by serving as a platform for the communities to exchange ideas on how we can fully take advantage of the visible hand of microstructure for the future development of related fields and disciplines.

\section{DECLARATIONS}

\section{Authors' contributions}

All authors contribute equally.

\section{Availability of data and materials}

Not applicable.

\section{Financial support and sponsorship}

None. 


\section{Conflicts of interest}

All authors declared that there are no conflicts of interest.

\section{Ethical approval and consent to participate}

Not applicable.

\section{Consent for publication}

Not applicable.

\section{Copyright}

(C) The Author(s) 2021. 